\title{
Bessel collocation approach for approximate solutions of Hantavirus infection model
}

\author{
Şuayip Yüzbasi \\ Department of Mathematics, Faculty of Science, Akdeniz University, Antalya, Turkey \\ Received: 29 November 2016, Accepted: 17 June 2017 \\ Published online: 11 November 2017.
}

\begin{abstract}
In this study, a collocation method is introduced to find the approximate solutions of Hantavirus infection model which is a system of nonlinear ordinary differential equations. The method is based on the Bessel functions of the first kind, matrix operations and collocation points. This method converts Hantavirus infection model into a matrix equation in terms of the Bessel functions of first kind, matrix operations and collocation points. The matrix equation corresponds to a system of nonlinear equations with the unknown Bessel coefficients. The reliability and efficiency of the suggested scheme are demonstrated by numerical applications and all numerical calculations have been done by using a program written in Maple.
\end{abstract}

Keywords: Hantavirus infection model, population model, Bessel collocation method, Bessel functions of first kind, numerical solution, nonlinear differential equation systems.

\section{Introduction}

Population model problems have an important place in Mathematical Biology. In recent years, some researchers have studied solutions of population model problems such as continuous population models [1,2], population growth model with fractional order [3,4], Volterra population model [5], HIV Infection models [6,7,8], population models of integro-differential equations [9,10,11], Hantavirus infection model [12,13,14], some model problems in Biology [15, 16]. Also, Akbar has studied sutterby fluid model for blood flow [17] and A. Sohail et al. have analysed fractional-order tumor model [18].

In this paper, by means of the matrix relations between the Bessel functions of first kind $J_{n}(x)$ and their derivatives, we apply the Bessel collocation method [19,20,21] to obtain approximate solutions of Hantavirus infection model studied in $[12,13,14,22,23]$. This model is characterized by a system of the nonlinear differential equations

$$
\begin{cases}\frac{d S}{d t}=b(S+I)-c S-\frac{S(S+I)}{k}-a S I & , S(0)=\lambda_{1}, I(0)=\lambda_{2}, 0 \leq t \leq R \\ \frac{d I}{d t}=-c I-\frac{I(S+I)}{k} & \end{cases}
$$

so that $S(t)$ and $I(t)$ are the populations of susceptible and infected mice, respectively, and $M(t)=S(t)+I(t)$ is the total population of mice.

The meanings of other terms in (1) are as follows.

Births: $b(S+I)$ shows the birth of susceptible newborn mice, all with a rate proportional to the total population, since all mice make contribution to breeding regardless of whether it is susceptible or infected. 
Deaths: $c$ denotes the rate of depletion by death for natural reasons, proportional to the corresponding density.

Competition: $-S I(S+I) / k$ indicates the process of limiting the increase of population due to conflict of resource sharing. $k$ shows that the carrying capacity of all means to protect the population. Higher values of $k$ represent good environmental conditions which are water, food, housing availability, favorable climatic conditions.

Infection: aSI is considered to be constant in a rapidly due to an encounter with an infectious mouse shows the number of infected susceptible mice.

In addition, $a, b, c$ and $k$ are parameters that characterize the system in (1). $k$ is chosen to be the control parameter of the dynamics because it best represents the influence of the environment. Although the system has four equilibriums, two of them are irrelevant to the analysis, that is, the null state (very unstable) and state $a$ with $I<0$ for any parameters. The other two equilibriums interchange their stability character at a critical value of the carrying capacity, which is given as

$$
k_{c}=\frac{b}{a(b-c)}
$$

Changes in ecological conditions and diversity of habitats can be observed by varying the value of $k$. A low value of $k$ means that resources are scarce and therefore the number of infected mice is lower. If $k<k_{c}$, the number of infected mice will reduce to zero as observed in field studies. However, when conditions improve again where $k>k_{c}$, the infection will reappear and may even show a drastic increase in the population of infected mice.

Our aim is to find approximate solutions of model (1) expressed in the truncated Bessel series form

$$
S(t)=\sum_{n=0}^{N} a_{1, n} J_{n}(t) \quad \text { and } \quad I(t)=\sum_{n=0}^{N} a_{2, n} J_{n}(t)
$$

so that $a_{1, n}$ and $a_{2, n}(n=0,1,2, \ldots, N)$ are the unknown Bessel coefficients and $J_{n}(t), n=0,1,2, \ldots, N$ are the Bessel functions of first kind defined by

$$
J_{n}(t)=\sum_{k=0}^{\llbracket \frac{N-n}{2} \rrbracket} \frac{(-1)^{k}}{k !(k+n) !}\left(\frac{t}{2}\right)^{2 k+n}, n \in \mathbb{N}, 0 \leq t<\infty .
$$

\section{Bessel collocation method for Hantavirus infection model}

Firstly, let us show model (1) in the form

$$
\left\{\begin{array}{l}
\frac{d S}{d t}=(b-c) S+b I-\frac{1}{k} S^{2}-\left(\frac{1}{k}+a\right) S I \\
\frac{d I}{d t}=-c I-\frac{1}{k} I^{2}-\left(\frac{1}{k}-a\right) S I
\end{array} .\right.
$$

We consider the desired solution $S(t)$ and $I(t)$ of system (5) defined by the truncated Bessel series (3). Now, we write the matrix form of the functions defined in relation (2) as

$$
S(t)=\mathbf{J}(t) \mathbf{A}_{1} \quad \text { and } \quad I(t)=\mathbf{J}(t) \mathbf{A}_{2}
$$

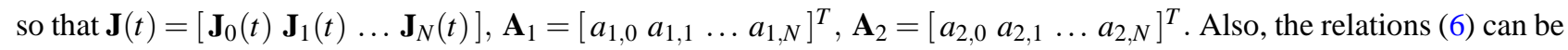
written in matrix form

$$
S(t)=\mathbf{T}(t) \mathbf{D}^{T} \mathbf{A}_{1} \quad \text { and } \quad I(t)=\mathbf{T}(t) \mathbf{D}^{T} \mathbf{A}_{2}
$$


where $\mathbf{T}(t)=\left[1 t t^{2} \ldots t^{N}\right]$ and if $N$ is odd,

$$
\mathbf{D}=\left[\begin{array}{cccccc}
\frac{1}{0 ! 0 ! 2^{0}} & 0 & \frac{-1}{1 ! 1 ! 2^{2}} & \cdots & \frac{(-1)^{\frac{N-1}{2}}}{\left(\frac{N-1}{2}\right) !\left(\frac{N-1}{2}\right) ! 2^{N-1}} & 0 \\
0 & \frac{1}{0 ! 1 ! 2^{1}} & 0 & \cdots & 0 & \frac{(-1)^{\frac{N-1}{2}}}{\left(\frac{N-1}{2}\right) !\left(\frac{N+1}{2}\right) ! 2^{N}} \\
0 & 0 & \frac{1}{0 ! 2 ! 2^{2}} & \cdots & \frac{(-1)^{\frac{N-3}{2}}}{\left(\frac{N-3}{2}\right) !\left(\frac{N+1}{2}\right) ! 2^{N-1}} & 0 \\
\vdots & \vdots & \vdots & \ddots & \vdots & \vdots \\
0 & 0 & 0 & \cdots & \frac{1}{0 !(N-1) ! 2^{N-1}} & 0 \\
0 & 0 & 0 & \cdots & 0 & \frac{1}{0 ! N ! 2^{N}}
\end{array}\right]_{(N+1) \times(N+1)}
$$

if $N$ is even,

$$
\mathbf{D}=\left[\begin{array}{cccccc}
\frac{1}{0 ! 0 ! 2^{0}} & 0 & \frac{-1}{1 ! 1 ! 2^{2}} & \cdots & 0 & \frac{(-1)^{\frac{N}{2}}}{\left(\frac{N}{2}\right) !\left(\frac{N}{2}\right) ! 2^{N}} \\
0 & \frac{1}{0 ! 1 ! 2^{1}} & 0 & \cdots & \frac{(-1)^{\frac{N-2}{2}}}{\left(\frac{N-2}{2}\right) !\left(\frac{N}{2}\right) ! 2^{N-1}} & 0 \\
0 & 0 & \frac{1}{0 ! 2 ! 2^{2}} & \cdots & 0 & \frac{(-1)^{\frac{N-2}{2}}}{\left(\frac{N-2}{2}\right) !\left(\frac{N+2}{2}\right) ! 2^{N}} \\
\vdots & \vdots & \vdots & \ddots & \vdots & \vdots \\
0 & 0 & 0 & \cdots & \frac{1}{0 !(N-1) ! 2^{N-1}} & 0 \\
0 & 0 & 0 & \cdots & 0 & \frac{1}{0 ! N ! 2^{N}}
\end{array}\right]_{(N+1) \times(N+1)}
$$

The relation between the matrix $\mathbf{T}(t)$ and its derivative $\mathbf{T}^{(1)}(t)$ is given by

$$
\mathbf{T}^{(1)}(t)=\mathbf{T}(t) \mathbf{B}^{T}
$$

where

$$
\mathbf{B}^{T}=\left[\begin{array}{ccccc}
0 & 1 & 0 & \ldots & 0 \\
0 & 0 & 2 & \ldots & 0 \\
\vdots & \vdots & \vdots & \ddots & \vdots \\
0 & 0 & 0 & \ldots & N \\
0 & 0 & 0 & \ldots & 0
\end{array}\right]
$$

By aid of the relations (7) and (8) we have recurrence relations

$$
S^{(1)}(t)=\mathbf{T}(t) \mathbf{B}^{T} \mathbf{D}^{T} \mathbf{A}_{1} \quad \text { and } \quad I^{(1)}(t)=\mathbf{T}(t) \mathbf{B}^{T} \mathbf{D}^{T} \mathbf{A}_{2} .
$$

Thus, we can express the matrices $\mathbf{y}(t)$ and $\mathbf{y}^{(1)}(t)$ as follows:

$$
\mathbf{y}(t)=\overline{\mathbf{T}}(t) \overline{\mathbf{D}} \mathbf{A} \quad \text { and } \quad \mathbf{y}^{(1)}(t)=\overline{\mathbf{T}}(t) \overline{\mathbf{B}} \overline{\mathbf{D}} \mathbf{A}
$$

so that $\mathbf{y}(t)=[S(t) I(t)]^{T}, \mathbf{y}^{(1)}(t)=\left[S^{(1)}(t) I^{(1)}(t)\right]^{T}, \overline{\mathbf{T}}(t)=\operatorname{diag}(\mathbf{T}(t) \mathbf{T}(t)), \overline{\mathbf{D}}=\operatorname{diag}\left(\mathbf{D}^{T} \mathbf{D}^{T}\right), \overline{\mathbf{B}}=\operatorname{diag}\left(\mathbf{B}^{T} \mathbf{B}^{T}\right)$ and $\mathbf{A}=\left[\begin{array}{ll}\mathbf{A}_{1} & \mathbf{A}_{2}\end{array}\right]^{T}$. 
Now, we can show the system (5) with the matrix form

$$
\mathbf{y}^{(1)}(t)=\mathbf{P y}(t)+\mathbf{K} \overline{\mathbf{y}}(t) \mathbf{y}(t)+\mathbf{L y}_{1,2}(t)
$$

where

$$
\mathbf{P}=\left[\begin{array}{cc}
b-c & b \\
0 & -c
\end{array}\right], \mathbf{K}=\left[\begin{array}{cc}
-1 / k & 0 \\
0 & -1 / k
\end{array}\right], \mathbf{L}=\left[\begin{array}{c}
-(1 / k)-a \\
-(1 / k)+a
\end{array}\right], \overline{\mathbf{y}}(t)=\left[\begin{array}{cc}
S(t) & 0 \\
0 & I(t)
\end{array}\right] \quad \text { and } \quad \mathbf{y}_{1,2}(t)=[S(t) I(t)]
$$

By using in Eq. (11) the collocation points defined by

$$
t_{i}=\frac{R}{N} i, i=0,1, \ldots, N
$$

we obtain the system of the matrix equations

$$
\mathbf{y}^{(1)}\left(t_{s}\right)=\mathbf{P y}\left(t_{s}\right)+\mathbf{K} \overline{\mathbf{y}}\left(t_{s}\right) \mathbf{y}\left(t_{s}\right)+\mathbf{L} \mathbf{y}_{1,2}\left(t_{s}\right)
$$

or briefly the fundamental matrix equation

$$
\mathbf{Y}^{(1)}-\overline{\mathbf{P}} \mathbf{Y}-\overline{\mathbf{K}} \overline{\mathbf{Y}} \mathbf{Y}-\overline{\mathbf{L}} \overline{\mathbf{Y}}=\mathbf{G}
$$

where $\quad \mathbf{Y}^{(1)}=\left[\mathbf{y}^{(1)}\left(t_{0}\right) \mathbf{y}^{(1)}\left(t_{1}\right) \ldots \mathbf{y}^{(1)}\left(t_{N}\right)\right]^{T}, r=1,2, \ldots, N+1, \overline{\mathbf{P}}=\operatorname{diag}\left(\mathbf{P}_{1}, \mathbf{P}_{2}, \ldots, \mathbf{P}_{N+1}\right), \mathbf{P}_{r}=\mathbf{P}, \mathbf{Y}=$ $\left[\mathbf{y}\left(t_{0}\right) \mathbf{y}\left(t_{1}\right) \ldots \mathbf{y}\left(t_{N}\right)\right]^{T}, \overline{\mathbf{K}}=\operatorname{diag}\left(\mathbf{K}_{1}, \mathbf{K}_{2}, \ldots, \mathbf{K}_{N+1}\right), \mathbf{K}_{r}=\mathbf{K}, \overline{\mathbf{Y}}=\operatorname{diag}\left(\overline{\mathbf{y}}\left(t_{0}\right), \overline{\mathbf{y}}\left(t_{1}\right), \ldots, \overline{\mathbf{y}}\left(t_{N}\right)\right), \overline{\mathbf{L}}=$ $\operatorname{diag}\left(\mathbf{L}_{1}, \mathbf{L}_{2}, \ldots, \mathbf{L}_{N+1}\right), \mathbf{L}_{r}=\mathbf{L}, \overline{\overline{\mathbf{Y}}}=\left[\mathbf{y}_{1,2}\left(t_{0}\right) \mathbf{y}_{1,2}\left(t_{1}\right) \ldots \mathbf{y}_{1,2}\left(t_{N}\right)\right]^{T}$ and $\mathbf{G}=\left[\begin{array}{llll}0 & 0 & \ldots & 0\end{array}\right]_{(2 N+1) \times 1}^{T}$. Using relations (10) and the collocation points (12), we have

$$
\mathbf{y}\left(t_{s}\right)=\overline{\mathbf{T}}\left(t_{s}\right) \overline{\mathbf{D}} \mathbf{A} \quad \text { and } \quad \mathbf{y}^{(1)}\left(t_{s}\right)=\overline{\mathbf{T}}\left(t_{s}\right) \overline{\mathbf{B}} \overline{\mathbf{D}} \mathbf{A}
$$

which can be written as

$$
\mathbf{Y}=\mathbf{T} \overline{\mathbf{D}} \mathbf{A} \quad \text { and } \quad \mathbf{Y}^{(1)}=\mathbf{T} \overline{\mathbf{B}} \overline{\mathbf{D}} \mathbf{A}
$$

where

$$
\mathbf{T}=\left[\overline{\mathbf{T}}\left(t_{0}\right) \overline{\mathbf{T}}\left(t_{1}\right) \ldots \overline{\mathbf{T}}\left(t_{N}\right)\right]^{T}, \overline{\mathbf{T}}\left(t_{s}\right)=\operatorname{diag}\left(\mathbf{T}\left(t_{s}\right) \quad \mathbf{T}\left(t_{s}\right)\right) \quad \text { and } \quad s=0,1, \ldots, N
$$

By aid of the collocation points (12) and the matrix given in Eq. (11), we have

$$
\overline{\mathbf{Y}}=\operatorname{diag}\left(\overline{\mathbf{y}}\left(t_{0}\right), \overline{\mathbf{y}}\left(t_{1}\right), \ldots, \overline{\mathbf{y}}\left(t_{N}\right)\right)=\operatorname{diag}\left(\overline{\mathbf{T}}\left(t_{0}\right) \overline{\mathbf{D}} \overline{\mathbf{A}}, \overline{\mathbf{T}}\left(t_{1}\right) \overline{\mathbf{D}} \overline{\mathbf{A}}, \ldots, \overline{\mathbf{T}}\left(t_{N}\right) \overline{\mathbf{D}} \overline{\mathbf{A}}\right)=\overline{\mathbf{T}} \overline{\overline{\mathbf{D}}} \overline{\overline{\mathbf{A}}}
$$

where $\quad \overline{\mathbf{T}}=\operatorname{diag}\left(\overline{\mathbf{T}}\left(t_{0}\right), \overline{\mathbf{T}}\left(t_{1}\right), \ldots, \overline{\mathbf{T}}\left(t_{N}\right)\right), \overline{\mathbf{T}}(t)=\operatorname{diag}(\mathbf{T}(t) \quad \mathbf{T}(t)), \overline{\overline{\mathbf{D}}}=\operatorname{diag}\left(\overline{\mathbf{D}}_{1}, \overline{\mathbf{D}}_{2}, \ldots, \overline{\mathbf{D}}_{N+1}\right), \overline{\mathbf{D}}_{r}=\overline{\mathbf{D}}_{2}$ $\overline{\mathbf{D}}=\operatorname{diag}\left(\mathbf{D}^{T} \quad \mathbf{D}^{T}\right), \overline{\overline{\mathbf{A}}}=\operatorname{diag}\left(\tilde{\mathbf{A}}_{1}, \tilde{\mathbf{A}}_{2}, \ldots, \tilde{\mathbf{A}}_{N+1}\right), \tilde{\mathbf{A}}_{r}=\tilde{\mathbf{A}}$ and $\tilde{\mathbf{A}}=\operatorname{diag}\left(\overline{\mathbf{A}}_{1} \quad \overline{\mathbf{A}}_{2}\right)$. Similarly, substituting the collocation points (12) into the $\mathbf{y}_{1,2}(t)$ in Eq. (11), we obtain the matrix representation

$$
\overline{\overline{\mathbf{Y}}}=\left[\mathbf{y}_{1,2}\left(t_{0}\right) \mathbf{y}_{1,2}\left(t_{1}\right) \ldots \mathbf{y}_{1,2}\left(t_{N}\right)\right]^{T}=\left[S\left(t_{0}\right) I\left(t_{0}\right) S\left(t_{1}\right) I\left(t_{1}\right) \ldots S\left(t_{N}\right) I\left(t_{N}\right)\right]^{T}=\overline{\mathbf{S}} \overline{\mathbf{I}}
$$

where

$$
\overline{\mathbf{S}}=\tilde{\mathbf{T}} \tilde{\mathbf{D}} \overline{\mathbf{A}}_{1} \quad \text { and } \quad \overline{\mathbf{I}}=\tilde{\tilde{\mathbf{T}}} \tilde{\tilde{\mathbf{D}}} \mathbf{A}
$$


so $\quad$ that $\quad \tilde{\mathbf{T}}=\operatorname{diag}\left(\mathbf{T}\left(t_{0}\right), \mathbf{T}\left(t_{1}\right), \ldots, \mathbf{T}\left(t_{N}\right)\right), r=1,2, \ldots, N+1, \tilde{\mathbf{D}}=\operatorname{diag}\left(\mathbf{D}_{1}, \mathbf{D}_{2}, \ldots, \mathbf{D}_{N+1}\right), \mathbf{D}_{r}=\mathbf{D}^{T}$, $\overline{\mathbf{A}}_{1}=\operatorname{diag}\left(\tilde{\tilde{\mathbf{A}}}_{1}, \tilde{\tilde{\mathbf{A}}}_{2}, \ldots, \tilde{\tilde{\mathbf{A}}}_{N+1}\right), \tilde{\tilde{\mathbf{A}}}_{r}=\mathbf{A}_{1}, \tilde{\tilde{\mathbf{T}}}=\left[\mathbf{T}\left(t_{0}\right) \mathbf{T}\left(t_{1}\right) \ldots \mathbf{T}\left(t_{N}\right)\right]^{T}, \tilde{\tilde{\mathbf{D}}}=\left[\begin{array}{ll}\mathbf{0} & \mathbf{D}^{T}\end{array}\right]$ and $\mathbf{0}=[0]_{(N+1) \times(N+1)}$. Substituting relations (14)-(17) into Eq. (13), we have fundamental matrix equation

$$
\left\{\mathbf{T} \overline{\mathbf{B}} \overline{\mathbf{D}}-\overline{\mathbf{P}} \mathbf{T} \overline{\mathbf{D}}-\overline{\mathbf{K}} \overline{\mathbf{T}} \overline{\overline{\mathbf{D}}} \overline{\overline{\mathbf{A}}} \mathbf{T} \overline{\mathbf{D}}-\overline{\mathbf{L}} \tilde{\mathbf{T}} \tilde{\mathbf{D}} \overline{\mathbf{A}}_{1} \tilde{\tilde{\mathbf{T}}} \tilde{\mathbf{D}}\right\} \mathbf{A}=\mathbf{G} .
$$

We can briefly write Eq. (18) in the form

$$
\mathbf{W A}=\mathbf{G} \quad \text { or } \quad[\mathbf{W} ; \mathbf{G}] ; \quad \mathbf{W}=\mathbf{T} \overline{\mathbf{B}} \overline{\mathbf{D}}-\overline{\mathbf{P}} \mathbf{T} \overline{\mathbf{D}}-\overline{\mathbf{K}} \overline{\mathbf{T}} \overline{\overline{\mathbf{D}}} \overline{\overline{\mathbf{A}}} \mathbf{T} \overline{\mathbf{D}}-\overline{\mathbf{L}} \tilde{\mathbf{T}} \tilde{\mathbf{D}} \overline{\mathbf{A}}_{1} \tilde{\tilde{\mathbf{T}}} \tilde{\tilde{\mathbf{D}}}
$$

which corresponds to a system of the $2(N+1)$ nonlinear algebraic equations with the unknown Bessel coefficients $a_{1, n}$ and $a_{2, n}, n=0,1,2, \ldots, N$.

By aid of the relation (10), the matrix form for conditions given in model (2) can be written as

$$
\mathbf{U A}=[\lambda] \quad \text { or }[\mathbf{U} ; \lambda]
$$

where $\mathbf{U}=\overline{\mathbf{T}}(0) \overline{\mathbf{D}} \mathbf{A}$ and $\lambda=\left[\begin{array}{l}\lambda_{1} \\ \lambda_{2}\end{array}\right]$.

Consequently, replacing two rows of the augmented matrix $[\mathbf{W} ; \mathbf{G}]$ by the rows of the matrix $[\mathbf{U} ; \lambda]$, we have the new augmented matrix

$$
[\tilde{\mathbf{W}} ; \tilde{\mathbf{G}}] \quad \text { or } \quad \tilde{\mathbf{W}} \mathbf{A}=\tilde{\mathbf{G}}
$$

which is a nonlinear algebraic system. The unknown Bessel coefficients can be found by solving this system. The unknown Bessel coefficients $a_{i, 0}, a_{i, 1}, \ldots, a_{i, 2},(i=1,2)$ is substituted in Eq. (6). Thus we obtain the Bessel polynomial solutions

$$
S_{N}(t)=\sum_{n=0}^{N} a_{1, n} J_{n}(t) \quad \text { and } \quad I_{N}(t)=\sum_{n=0}^{N} a_{2, n} J_{n}(t)
$$

\section{Accuracy of the solutions}

Firstly, let us check the accuracies of the solutions (22) as follows.

Since the truncated Bessel series (22) are approximate solutions of system (1), when the functions $S_{N}(t), I_{N}(t)$ and their derivatives are substituted in system (1), the resulting equation must be satisfied approximately; that is, for $t=t_{q} \in[0, b], q=0,1,2, \ldots$

$$
\left\{\begin{array}{l}
E_{1, N}\left(t_{q}\right)=\left|S^{(1)}\left(t_{q}\right)-b\left(S\left(t_{q}\right)+I\left(t_{q}\right)\right)+c S\left(t_{q}\right)+\frac{S\left(t_{q}\right)\left(S\left(t_{q}\right)+I\left(t_{q}\right)\right)}{k}+a S\left(t_{q}\right) I\left(t_{q}\right)\right| \cong 0, \\
E_{2, N}\left(t_{q}\right)=\left|I^{(1)}\left(t_{q}\right)+c I\left(t_{q}\right)+\frac{I\left(t_{q}\right)\left(S\left(t_{q}\right)+I\left(t_{q}\right)\right)}{k}-a S\left(t_{q}\right) I\left(t_{q}\right)\right| \cong 0
\end{array}\right.
$$

and $E_{i, N}\left(t_{q}\right) \leq 10^{-k_{q}}, i=1,2\left(k_{q}\right.$ positive integer).

If $\max 10^{-k_{q}}=10^{-k}$ ( $k$ positive integer) is prescribed, then the truncation limit $N$ is increased until the difference $E_{i, N}\left(t_{q}\right)$ at each of the points becomes smaller than the prescribed $10^{-k}$, see $[2,14,24]$. 


\section{Numerical application}

In this section, we apply the method presented in interval $0 \leq t \leq 3$ for solving Hantavirus infection model [12,13, 14,22, 23]. We consider the model (1) for $a=0.1, b=1, c=0.5, k=20<k_{c}$ with the initial conditions $S(0)=10$ and $I(0)=10$. By applying the method in Section 2 for $N=5$ and $N=8$, we obtain the approximate solutions

$$
\left\{\begin{array}{l}
S_{5}(t)=10-5 t+2.53936620660 t^{2}-3.06990096594 t^{3}+1.20390757509 t^{4}-(0.78748587762 e-1) t^{5} \\
I_{5}(t)=10-5 t+2.34588523040 t^{2}-.221683403260 t^{3}-0.188484008271 t^{4}+(0.255542650706 e-1) t^{5}
\end{array}\right.
$$

and

$$
\left\{\begin{aligned}
S_{8}(t)= & 10-5 t+3.94977835745 t^{2}-4.24915316010 t^{3}+1.46442274516 t^{4}-(0.5019073487 e-2) t^{5} \\
& -0.147998114822 t^{6}+(0.552734255182 e-1) t^{7}-(0.673569602508 e-2) t^{8} \\
I_{8}(t)= & 10-5 t+1.99844138491 t^{2}-(0.47712783635 e-1) t^{3}-(0.72719454610 e-1) t^{4}-(0.347519722572 e-1) t^{5} \\
& +(0.598016780188 e-2) t^{6}+(0.136711371338 e-2) t^{7}-(0.183196494882 e-3) t^{8}
\end{aligned}\right.
$$

We display a plot of the approximate solutions $S_{N}(t)$ obtained by the present method for $N=5,8$ in Figure $1 \mathrm{a}$. Figure $1 \mathrm{~b}$ show a plot of the approximate solutions $I_{N}(t)$ obtained by the present method for $N=5,8$. We give the error functions obtained with accuracy (22) of the solution obtained by the present method for $N=5,8$ in Figure 2 a. Figure 2 b) denotes the error functions obtained with accuracy (22) of the solution obtained by the present method for $N=5,8$. Figure 1a shows that the population of suscepted mice $S(t)$ will approach to 8 for $N=5,8$. Figure 1 b displays that the population of infected mice $I(t)$ will decease to 3.5 for $N=5,8$.

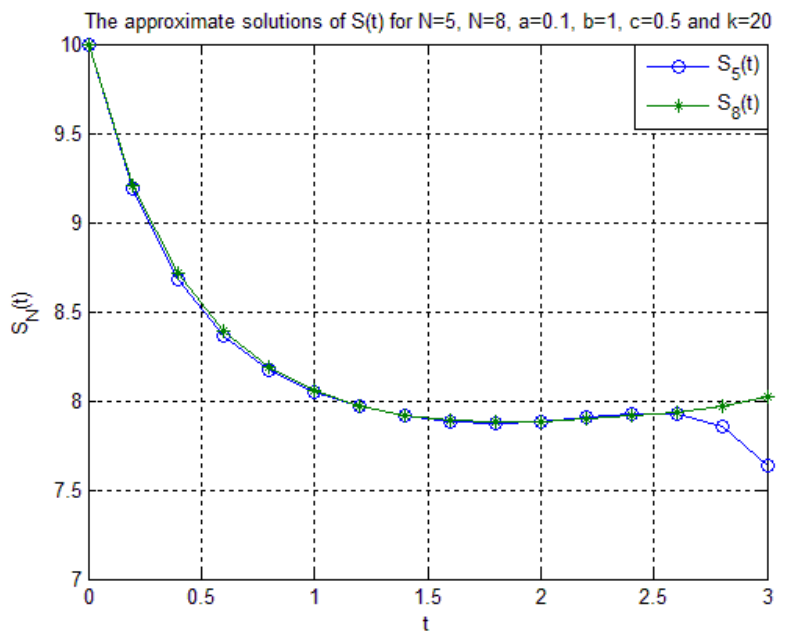

(a) Graph of the solutions $S_{N}(t)$ for $N=5,8$.

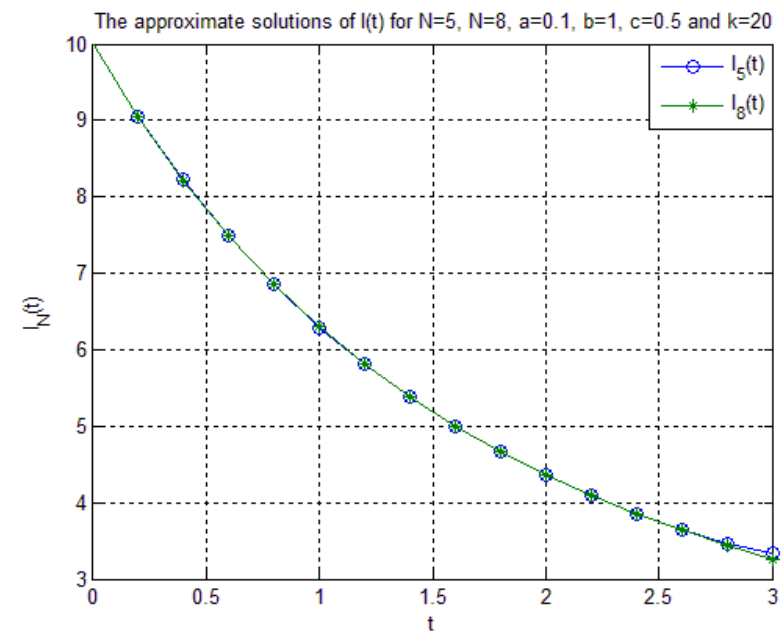

(b) Graph of the solutions $I_{N}(t)$ for $N=5,8$.

Fig. 1 


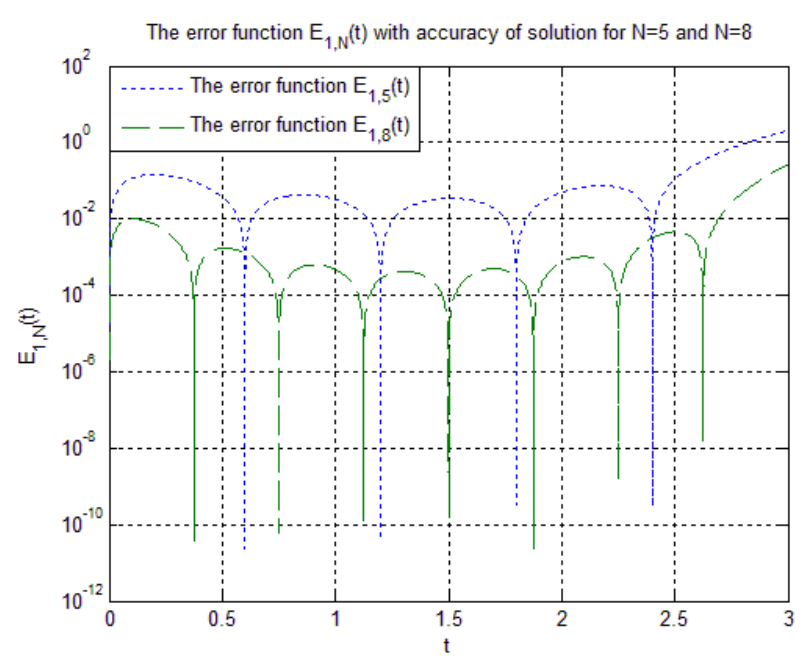

(a) Graph of the error functions obtained with accuracy of the solution $S_{N}(t)$ for $N=5,8$.

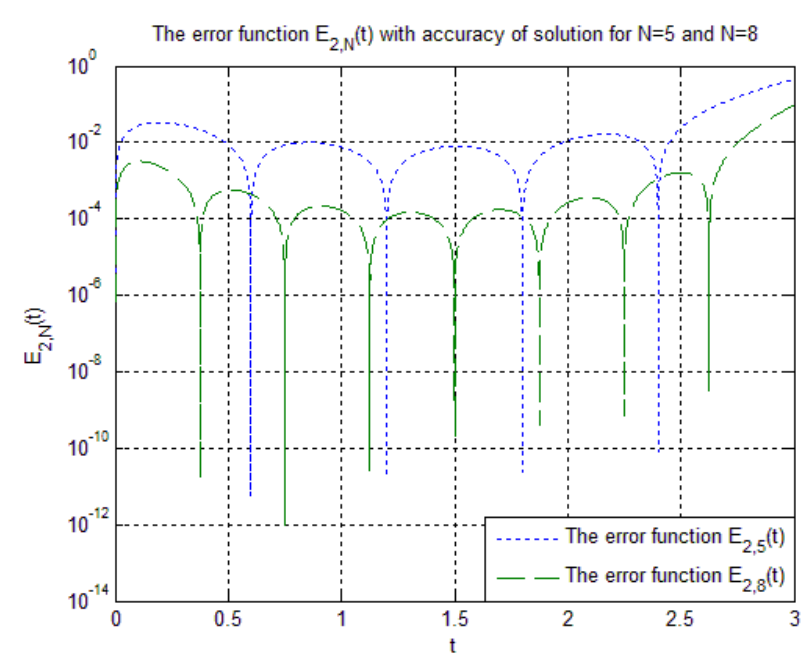

(b) Graph of the error functions obtained with accuracy of the solution $I_{N}(t)$ for $N=5,8$.

Fig. 2

\section{Conclusions}

In this study, the Bessel collocation method has been presented for solving Hantavirus infection model which is a system of nonlinear ordinary differential equations. We demonstrated the accuracy and efficiency of this method with an example. We assured the correctness of the obtained solutions by putting them back into the original equation with the aid of Maple; it provides an extra measure of confidence in the results. The computations associated with the example have been performed using a computer code written in Maple 9. The basic idea described in this paper is expected to be further employed to solve other similar nonlinear problems.

\section{Acknowledgements}

The authors are supported by the Scientific Research Project Administration of Akdeniz University.

\section{Competing interests}

The authors declare that they have no competing interests.

\section{Authors' contributions}

All authors have contributed to all parts of the article. All authors read and approved the final manuscript.

\section{References}

[1] S. Pamuk, The decomposition method for continuous population models for single and interacting species, Appl. Math. Comput. 163 (2005) 79-88. 
[2] Ş. Yüzbaşı, Bessel collocation approach for solving continuous population models for single and interacting species, Appl. Math. Model. 36(8) (2012) 3787-3802.

[3] H. Xu, Analytical approximations for a population growth model with fractional order, Commun. Nonlinear Sci. Numer. Simulat. 14 (2009) 1978-1983.

[4] S. Momani, R. Qaralleh, Numerical approximations and Padé approximants for a fractional population growth model, Appl. Math. Model. 31 (2007) 1907-1914.

[5] K.G. TeBeest, Numerical and analytical solutions of Volterra's population model, SIAM Rev. 39 (1997) 484-93.

[6] A. Gökdoğan, A. Yıldırım, M. Merdan, Solving a fractional order Model of HIV Infection of CD4+ T Cells, Math. Comput. Model. 54(9-10) (2011) 2132-2138.

[7] A. Mastroberardino, Y. Cheng, A. Abdelrazec, H. Liu, Mathematical modeling of the HIV/AIDS epidemic in Cuba, Int. J. Biomath. 8(4) (2015) 1550047.

[8] M. Merdan, Homotopy perturbation method for solving a model for HIV infection of CD4+ T cells, Istanb. Commerce Uni. J. Sci. 12 (2007) 39-52.

[9] Ş. Yüzbaşı, M. Sezer, Bayram Kemanc1, Numerical solutions of integro-differential equations and application of a population model with an improved Legendre method, Appl. Math. Model., 37(4) (2013) 2086-2101.

[10] F. Shakeri, M. Dehghan, Solution of a model describing biological species living together using the variational iteration method, Math. Comput. Model. 48 (2008) 685-699.

[11] Ş. Yüzbaş1, M. Sezer, An exponential approach for the system of nonlinear delay integro-differential equations describing biological species living together, Neural Comput. Appl. 27(3) (2016) 769-779.

[12] G. Abramson, V.M. Kenkre, Spatiotemporal patterns in the hantavirus infection, Phys. Rev. E Stat. Nonlin. Soft Matter Phys. 66 (2002) 011912.

[13] G. Abramson, V.M. Kenkre, T.L. Yates, B.R. Parmenter, Traveling waves of infection in the Hantavirus epidemics, Bull. Math. Biol. 65 (2003) 519-534.

[14] Ş. Yüzbaşı, M. Sezer, An exponential matrix method for numerical solutions of Hantavirus infection model, Appl. Appl. Math. 8(1) (2013) 99-115.

[15] R.C. Mittal, R. Jiwari, A Higher Order Numerical Scheme for Some Nonlinear Differential Equations: Models in Biology, Int. J. Comput. Methods Eng. Sci Mech. 12(3) (2011) 134-140.

[16] Y. Khan, H.Vázquez-Leal, Q. Wu, An efficient iterated method for mathematical biology model, Neural Comput. Appl. 23 (2013) 677-682.

[17] N.S. Akbar, Biomathematical study of Sutterby fluid model for blood flow in stenosed arteries, Int. J. Biomath. 8(6) (2015) 1550075.

[18] A. Sohail, S. Arshad, S. Javed, K. Maqbool, Numerical analysis of fractional-order tumor model, Int. J. Biomath. 8(5) (2015) 1550069.

[19] Ş. Yüzbaşı, N. Şahin, M. Sezer, Numerical solutions of systems of linear Fredholm integro-differential equations with Bessel polynomial bases. Comput. Math. Appl. 61 (2011) 3079-3096.

[20] Ş. Yüzbaşı, A numerical approach for solving a class of the nonlinear Lane-Emden type equations arising in astrophysics, Math. Meth. Appl. Sci. 34 (2011) 2218-2230.

[21] Ş. Yüzbaşı, N. Şahin, M. Sezer, A collocation approach for solving modelling the pollution of a system of lakes, Math. Comput. Model. 55 (2012) 330-341.

[22] S.M. Goh, A.I.M. Ismail, M.S.M. Noorani, I Hashim, Dynamics of the Hantavirus infection through variational iteration method. Nonlinear Anal.-Real. 10 (2009) 2171-2176.

[23] A. Gökdoğan, M. Merdan, A Yıldırım, A multistage differential transformation method for approximate solution of Hantavirus infection model, Commun. Nonlinear Sci. 17 (2012) 1-8.

[24] N. Kurt, M. Çevik, Polynomial solution of the single degree of freedom system by Taylor matrix method, Mech. Res. Commun. 35 (2008) 530-536. 